\title{
Effect of non-mesogenic chiral terphenylate on the formulation of room temperature ferroelectric liquid crystal mixtures suitable for display applications
}

\author{
Asim Debnath ${ }^{1}$, Pradip Kumar Mandal ${ }^{1 *}$, Abhisakh Sarma ${ }^{2}$, and Olof Gutowski ${ }^{2}$ \\ ${ }^{1}$ Department of Physics, University of North Bengal, Siliguri, India-734013 \\ ${ }^{2}$ Deutches Electronen-Synchrotron DESY, 22607 Hamburg, Germany \\ * Corresponding author. Email address: mandalpk.phys@ nbu.ac.in
}

\begin{abstract}
By varying the concentration of a chiral terphenylate dopant in a four-component achiral phenyl pyrimidine-based host mixture we have formulated six ferroelectric liquid crystal mixtures. Though the dopant is nonmesogenic it is observed that only $2 \mathrm{wt} \%$ of it induces polarity in the host mixture at $32^{\circ} \mathrm{C}$. With increasing concentration, the mixtures show ferroelectric behaviour even below ambient temperature down to at least $12^{\circ} \mathrm{C}$. Optical polarizing microscopy, differential scanning calorimetry, dielectric and electrooptic studies reveal that with respect to temperature range, phase sequence, spontaneous polarization, optical tilt angle and switching time the formulated mixtures are suitable for ferroelectric based liquid crystal displays.
\end{abstract}

Keywords: room temperature ferroelectric mixture; optical tilt; X-ray tilt; spontaneous polarization; switching time

\section{Introduction}

A material is said to be ferroelectric if it possesses a spontaneous polarization in absence of an external field and if its polarization is switched between $+v e$ and -ve states when the applied electric field is reversed. In general, some special types of solid materials which have non-centrosymmetric space group show ferroelectricity [1,2]. But, way back in 1974, using elegant symmetry 
considerations, Robert Meyer showed that ferroelectric properties could also be exhibited by fluid materials like tilted smectic liquid crystals if composed of chiral polar molecules $[3,4]$. Due to the reduced symmetry, SmC* phase possesses a spontaneous polarization $\left(\mathrm{P}_{\mathrm{S}}\right)$ in absence of an external electric field and on the application of an electric field the molecules switch around an imaginary cone such that the sign of the $\mathrm{P}_{\mathrm{S}}$ gets reversed. The sign of the $\mathrm{P}_{\mathrm{S}}$ can again be reversed to the initial state when an electric field of opposite direction is applied. Such type of switching of the molecules from one orientation to another in presence of electric field simultaneously changes the optical effect of the materials and mainly this electro-optical effect is employed in ferroelectric liquid crystal based display devices. With the help of this switching mechanism, Clark and Lagerwall first reported an ferroelectric display device which is known as surface stabilized ferroelectric liquid crystal (SSFLC) display device [5]. Subsequent interest in this field has increased tremendously in the last few decades. Several parameters such as temperature range, switching time, spontaneous polarization, tilt angle, length of the pitch, viscosity, etc are important characteristics of such materials. In order to achieve the desired properties of a display device one has to optimize all these parameters. But a single compound cannot satisfy all such requirements and hence multicomponent liquid crystal mixtures are usually formulated to optimize all the required properties necessary for display applications. Generally, a multicomponent mixture consists of two main constituents viz, achiral host mixture and a chiral dopant. The host mixture is usually a mixture of several achiral compounds with a reasonably wide SmC phase temperature range starting from room temperature or below, which controls the overall temperature range and tilt angle of the final mixture. On the other hand, the dopant is a compound with one or two chiral centers which controls the spontaneous polarization, viscosity, switching speed and the helical pitch of the final mixture [6-10]. Keeping all these things in mind we have prepared a four-component pyrimidine-based 
achiral host mixture (HM) which after doping with a non-mesogenic chiral dopant (NMC) resulted in several room temperature ferroelectric liquid crystal mixtures.

In this article, we report how the concentration of the NMC dopant influences the mesophase behaviour and the physical properties critically important for electro-optical properties of the display devices. It is observed that when only $2 \mathrm{wt} \%$ of $\mathbf{N M C}$ dopant is added to the $\mathbf{H M}$ it induces $\mathrm{SmC}^{*}$ phase at about $32^{\circ} \mathrm{C}$ and with increased concentration it exhibits $\mathrm{SmC}^{*}$ phase below ambient temperature down to at least $12^{\circ} \mathrm{C}$. These mixtures are found to possess high spontaneous polarization and relatively fast switching time which can be used in liquid crystal display devices.

\section{Experimental Techniques}

For the preparation of the host mixture HM we used four achiral phenyl pyrimidine compounds having tilted SmC phase. Molecular structures, transition temperatures and composition of the mixing components are shown in Table 1. Dopant NMC is a non-mesogenic protonated chiral terphenyl compound with chiral centres at opposite ends and it has a high melting point $\left(79.4^{\circ} \mathrm{C}\right)$. Molecular structure and transition temperature of the dopant are shown in Table 2. Final mixtures were prepared by adding various wt $\%$ of the NMC dopant in the host mixture (HM). To make them homogeneous the above mixtures were dissolved in chloroform and ultrasonicated at $65^{\circ} \mathrm{C}$ until evaporation of chloroform was completed. 
Table 1. Molecular structures and transition temperatures of the components and phase sequence of the host mixture

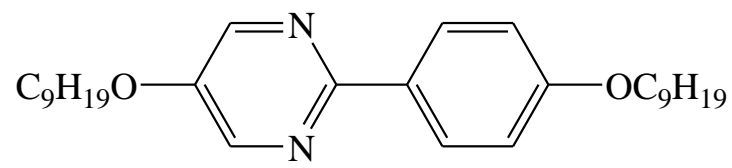

H1: $\mathrm{Cr} 61.8^{\circ} \mathrm{C} \mathrm{SmC} 95.6^{\circ} \mathrm{C} \mathrm{SmA} 99.1^{\circ} \mathrm{C} \mathrm{I}$

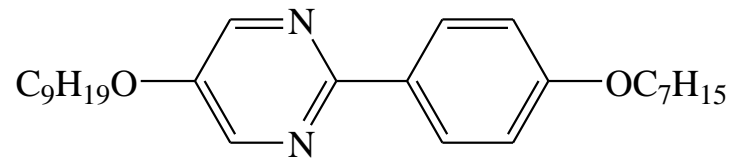

H2: $\mathrm{Cr} 57.4^{\circ} \mathrm{C} \operatorname{SmC} 95.1^{\circ} \mathrm{C} \operatorname{SmA} 98.4^{\circ} \mathrm{C} \mathrm{I}$

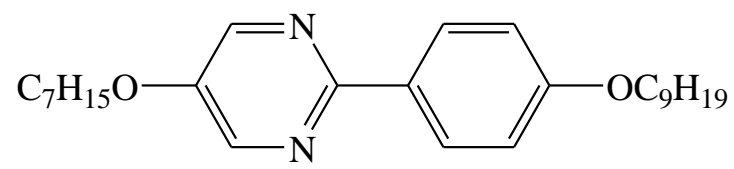

H3: $\mathrm{Cr} 57.2^{\circ} \mathrm{C} \operatorname{SmC} 79.1^{\circ} \mathrm{C} \mathrm{SmA} 91.2^{\circ} \mathrm{C} \mathrm{N} 94.7^{\circ} \mathrm{C} \mathrm{I}$

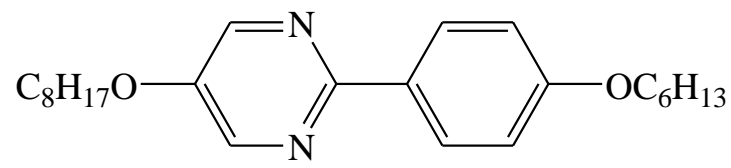

H4: $\mathrm{Cr} 27.5^{\circ} \mathrm{C} \mathrm{SmC} 46.3^{\circ} \mathrm{C} \mathrm{SmA} 57.5^{\circ} \mathrm{C} \mathrm{N} 65.6^{\circ} \mathrm{C} \mathrm{I}$

\section{Host Mixture (HM)}

Composition (wt $\%)$

Phase sequences and transition temperatures

H1: H2 : H3 : H4 :: $20: 20: 20: 40$

$\mathrm{Cr} 19^{\circ} \mathrm{C} \mathrm{SmC} 69.4^{\circ} \mathrm{C} \mathrm{SmA} 78.7^{\circ} \mathrm{C} \mathrm{N} 81.2^{\circ} \mathrm{C} \mathrm{I}$

Table 2. Molecular structure and transition temperature $\left({ }^{\circ} \mathrm{C}\right)$ of the $\mathbf{N M C}$ dopant Sample Molecular structure and transition temperature

NMC

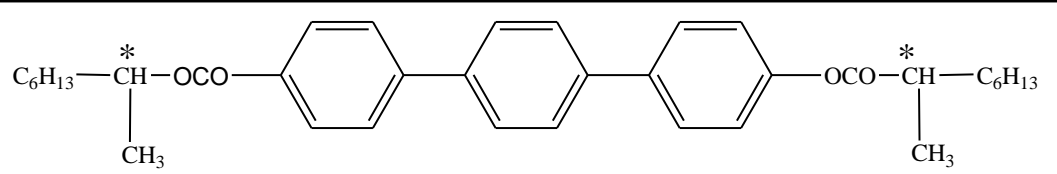

Cr 79.4 Iso

Polyimide-coated homogeneous (HG) dielectric cells, made of low resistive (about $10 \Omega /$ sq.) transparent indium tin oxide (ITO) coated electrodes having an effective area of $1 \mathrm{~cm}^{2}$ and a cell gap of $5 \mu \mathrm{m}$, were used for optical polarizing 
microscopy, dielectric, electro-optic, and synchrotron diffraction study. Cells were filled by capillary action with samples in isotropic state and to get proper alignment of the samples within the cell very slow regulated cooling was made.

Phase behavior and transition temperatures of the compounds and their mixtures were investigated by studying their topological defect structures (textures) under an Olympus BX41 polarizing microscope (OPM) equipped with a CCD camera. Using a Mettler FP90 temperature controller and FP82HT hot stage temperature of the samples was regulated with an accuracy of $\pm 0.1^{\circ} \mathrm{C}$. All the observations were made during heating at the rate of $0.2^{\circ} \mathrm{C} / \mathrm{min}$. Phase transition temperatures had also been determined by differential scanning calorimeter (DSC) using the same temperature controller but with different hot stage FP84HT and the FP99A software. During DSC measurements heating rate was taken as $5 \%$ min.

Structural investigation of the phases was made using PETRA III synchrotron beamline P07 at Physics Hutch station (EH2) at DESY, Hamburg. Synchrotron

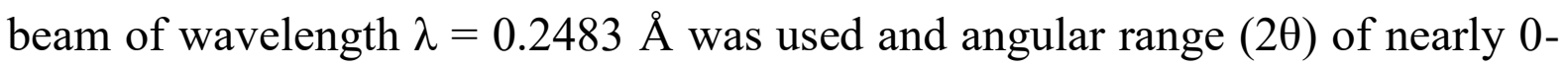
$3^{\circ}$ was covered. To avoid large absorption by the glass plates of the cell in normal geometry, the synchrotron beam was made to pass through the $5 \mu \mathrm{m}$ cell gap keeping parallel to the cell surfaces. A Perkin Elmer 2D detector was used for data collection. The total size of the detector was $400 \times 400 \mathrm{~mm}^{2}$ with individual pixel size $200 \times 200 \mu \mathrm{m}^{2}$ and it was placed $3.3 \mathrm{~m}$ away from the sample. QXRD program of G Jennings (version 0.9.8, 64 bit) was used for data acquisition. The same program was also used for analyzing the images. Images were integrated using a step size of $0.001^{\circ}$ to get the diffraction patterns (intensity versus Bragg angle 20). In this experiment, cell temperature was regulated with a LakeShore 340 temperature controller. Layer spacings were calculated using Bragg's relation; the peak positions were determined by fitting a Lorentzian function to intensity profile data. 
Complex dielectric permittivity of the mixtures was measured using a Hioki 353250 impedance analyzer in the frequency range from $50 \mathrm{~Hz}$ to $5 \mathrm{MHz}$, and automatic data acquisition arrangement was made using RS 232 interfacing with a PC.

Spontaneous polarization $\left(\mathrm{P}_{\mathrm{S}}\right)$ was measured as a function of temperature by the reversal current method [11] using a triangular wave $(5-70 \mathrm{Vpp}, 10 \mathrm{~Hz})$ obtained from an Agilent 3320A function generator. A digital storage oscilloscope (Tektronix TDS 2012B) was used to record the voltage drop (V) across a resistor $(\mathrm{R})$ in series with the cell as a function of time. The area under the curve was determined from the stored image after creating an appropriate baseline using commercial software. $\mathrm{P}_{\mathrm{S}}$ was calculated using the expression

$$
P_{S}=\frac{\int V d t}{2 A R}
$$

where $\mathrm{A}$ is the active area of the cell.

Measured $\mathrm{P}_{\mathrm{S}}$ data were fitted to the mean-field equation [12]

$$
P_{S}=P_{0}\left(1-T / T_{C}\right)^{\beta}
$$

to extract the value of the critical exponent $(\beta)$ for the secondary order parameter $\mathrm{P}_{\mathrm{S}}$ and the $\mathrm{SmC}^{*}-\mathrm{SmA}^{*}$ transition temperature $\left(\mathrm{T}_{\mathrm{C}}\right)$.

Tilt angle $(\theta)$, the angle made by the molecular long axis with the layer normal is the primary order parameter of ferroelectric $\mathrm{SmC}^{*}$ phase while $\mathrm{P}_{\mathrm{S}}$ is the secondary order parameter which is an outcome of the tilt. In this study, tilt angle $\theta$ was measured by electro-optical $\left(\theta_{\text {opt }}\right)$ method as well as by X-ray $\left(\theta_{\mathrm{X}}\right.$ ray) diffraction technique. On the application of an external ac field the

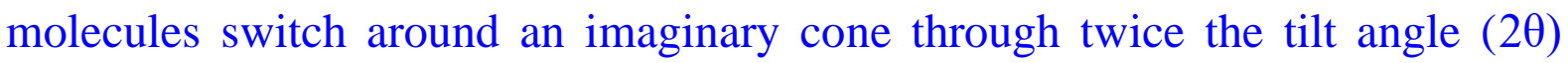
thereby reversing the sign of the spontaneous polarization. So the tilt of the molecules in smectic layers $\left(\theta_{\text {opt }}\right)$ was determined by measuring the angle of 
rotation of the FLC cell between two extinction conditions viewed between crossed polarisers under a microscope while observing switching of the molecules under a square pulse (magnitude 20-60 $\mathrm{V}_{\mathrm{pp}}$ ) of very low frequency $(10 \mathrm{mHz})$ [13]. On the other hand, the X-ray tilt was calculated from the measured layer spacings using the relation

$$
\theta_{X-r a y}=\cos ^{-1}\left(\frac{d_{C}}{d_{A}^{\max }}\right)
$$

where $d_{C}$ is the layer spacing in $S m C^{*}$ phase and $d_{A}{ }^{\text {max }}$ is the maximum layer spacing observed in $\mathrm{SmA}^{*}$ phase.

Response time was also measured by observing the switching of the molecules under a square pulse in the same dielectric cell. When a square wave pulse (20$60 \mathrm{Vpp}, 10 \mathrm{~Hz}$ ) was applied to the cell, a polarization bump occurred away on the time scale from the square pulse edge of the applied voltage. Measurement of the delay in the time provided the response time $(\tau)$ of the sample. For accurate measurement of $\tau$, oscilloscope data were stored in a computer and were analyzed by commercial software.

Rotational viscosities of the mixtures were calculated from the measured $\mathrm{P}_{\mathrm{S}}$ and $\tau$ using the relation [14]

$$
\gamma_{\varphi}=\tau P_{S} E
$$

where $\mathrm{E}$ was the applied electric field.

Estimated errors in optical tilt angle, spontaneous polarization and response time measurements were found to be nearly $\pm 0.1^{\circ}, \pm 0.5 \mathrm{nC} / \mathrm{cm}^{2}$ and $\pm 1 \mu \mathrm{s}$, respectively. 
Table 3. Composition, phase sequences, transition temperatures $\left({ }^{\circ} \mathrm{C}\right)$ and thermal range of $\mathrm{SmC}^{*}$ phase $(\Delta \mathrm{T})$ of the mixtures obtained from OPM and DSC studies. Transition temperatures from DSC are shown within parentheses.

\begin{tabular}{|c|c|c|c|}
\hline Mixture & Composition (wt\%) & Transition temperatures & $\Delta \mathrm{T}\left({ }^{\circ}\right)$ \\
\hline M1 & HM : NMC :: $98: 2$ & $\begin{array}{l}\text { Cr 32.0 SmC* } 88 \mathrm{SmA}^{*} 96.2 \mathrm{~N} * 97 \mathrm{I} \\
(30.9) \quad(87.3) \\
\text { (95.7) }\end{array}$ & 56 \\
\hline M2 & HM : NMC :: $95: 5$ & $\begin{array}{c}\mathrm{Cr}<12 \mathrm{SmC}^{*} 87.8 \mathrm{SmA}^{*} 95.8 \mathrm{~N}^{*} 96.3 \mathrm{I} \\
(88.8) \quad(94.3) \quad(97.8)\end{array}$ & 70.8 \\
\hline M3 & HM : NMC :: $90: 10$ & $\begin{array}{c}\mathrm{Cr}<12 \mathrm{SmC}^{*} 87.5 \mathrm{SmA}^{*} \text { 95.0 I } \\
(88.4)\end{array}$ & 70.5 \\
\hline M4 & HM : NMC :: $85: 15$ & $\begin{array}{c}\mathrm{Cr}<12 \mathrm{SmC}^{*} 87.4 \mathrm{SmA} * 93.7 \mathrm{I} \\
(88.1)\end{array}$ & 70.4 \\
\hline M5 & HM : NMC :: $80: 20$ & $\begin{array}{c}\mathrm{Cr}<12 \mathrm{SmC}^{*} 86.3 \mathrm{SmA}^{*} \text { 91.8 I } \\
(86.7) \quad(91.9)\end{array}$ & 70.3 \\
\hline M6 & HM : NMC :: $75: 25$ & $\begin{array}{c}\mathrm{Cr}<12 \mathrm{SmC}^{*} 85.6 \mathrm{SmA}^{*} 90.6 \mathrm{I} \\
(85.8)\end{array}$ & 68.6 \\
\hline
\end{tabular}

\section{Results and Discussions}

\subsection{OPM and DSC Study}

Phase behavior and transition temperatures observed from OPM as well as from DSC study during the heating cycle are shown in Table 3. Phase diagram and the DSC thermograms of the mixtures showing the variation of heat flow with temperature are shown in Fig. 1(a-c). It is observed that though the NMC itself is a non-mesogenic compound with a high melting point but when only 2 wt\% of it was doped in the HM it induced polarity (mixture M1) and exhibited ferroelectric $\mathrm{SmC}^{*}$ phase close to room temperature $\left(\sim 32{ }^{\circ} \mathrm{C}\right)$. In addition to SmC* phase, the mixture M1 was also found to exhibit SmA* and $\mathrm{N}^{*}$ phases before going to isotropic phase. At higher doping concentrations, the mixtures (M2-M6) exhibited $\mathrm{SmC}^{*}$ phase below ambient temperature down to at least $12^{\circ} \mathrm{C}$ (below which we could not measure). It was also noticed that with the increase of doping concentration, the $\mathrm{SmC}^{*}-\mathrm{SmA}^{*}$ transition temperature 
decreased slightly (that is the thermal range of the $\mathrm{SmC}^{*}$ phase decreased) and the cholesteric phase disappeared above $5 \mathrm{wt} \%$ of NMC.
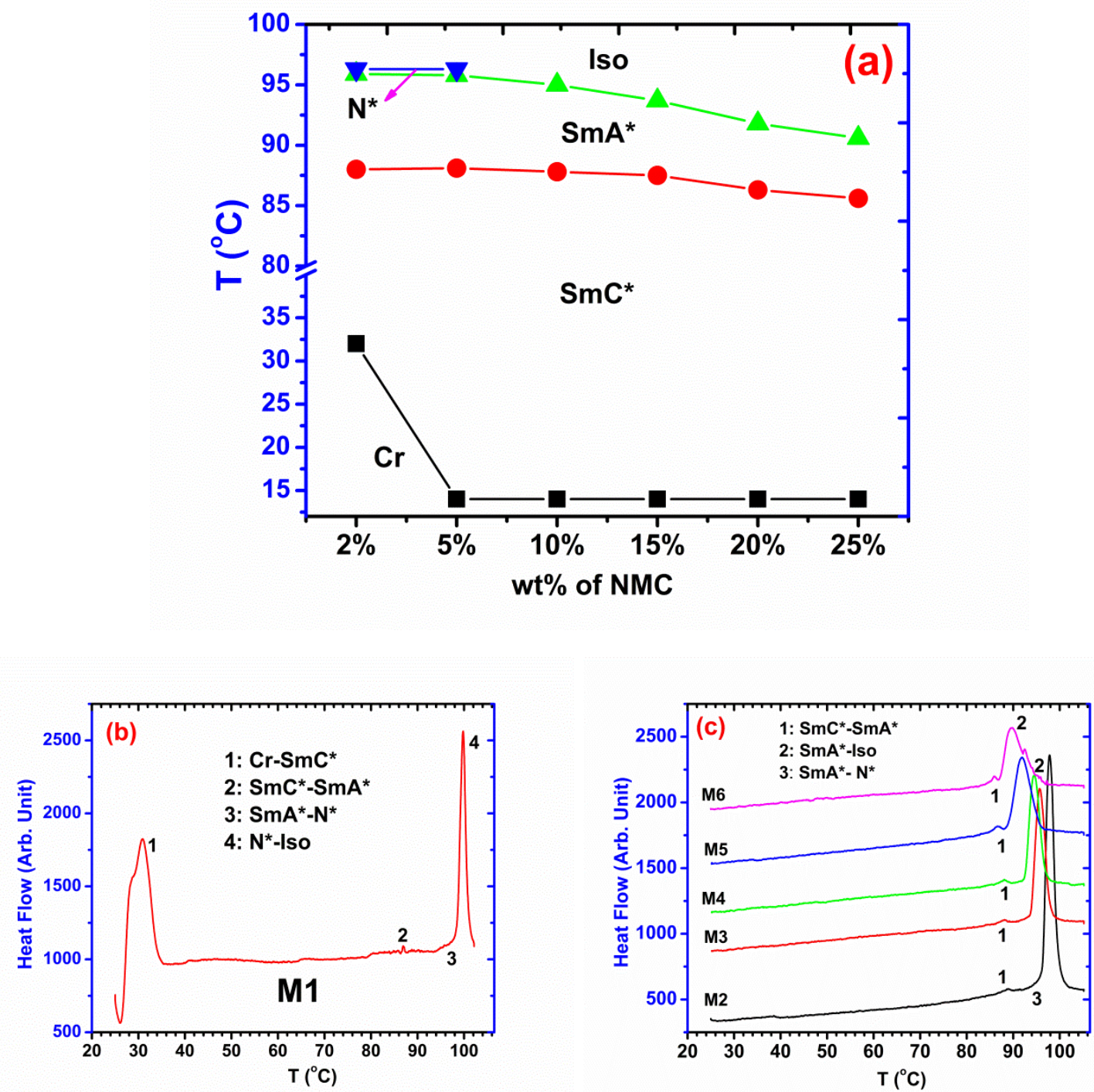

Fig. 1: (a) Phase diagram of the mixtures; DSC thermograms of (b) M1 and (c) M2, M3, M4, M5 and M6 during the heating cycle at $5^{\circ} \mathrm{C} / \mathrm{min}$

As a representative example, textures of the mixture M1 obtained under planar anchoring condition are shown in Fig. 2(a-c). Typical domain texture was observed in SmC* phase which transformed into a uniform planar aligned texture in $\mathrm{SmA}^{*}$ phase to a usual long pitch cholesteric droplets texture in $\mathrm{N}^{*}$ phase before going to isotropic phase [15]. 


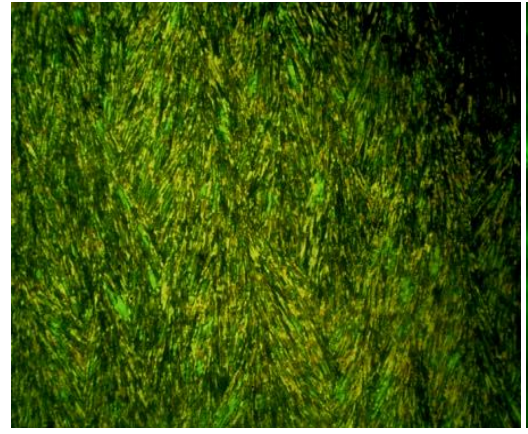

(a) $\mathrm{SmC}^{*}\left(35^{\circ} \mathrm{C}\right)$

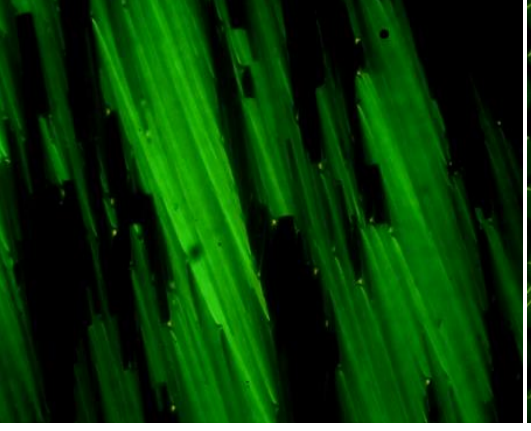

(b) $\mathrm{SmA}^{*}\left(90^{\circ} \mathrm{C}\right)$

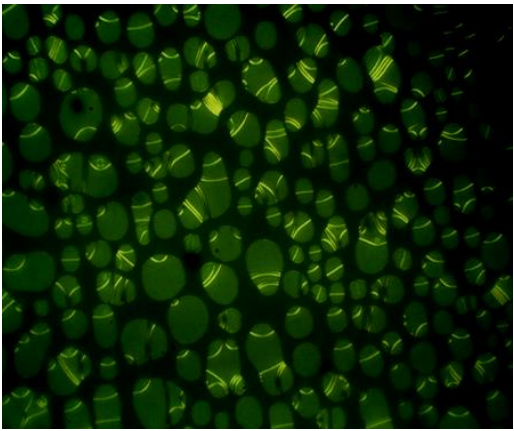

(c) $\mathrm{N}^{*}\left(96.5^{\circ} \mathrm{C}\right)$

Fig. 2: Observed textures of the mixture M1

It might be mentioned here that when four fluorinated chiral dopants differing only in number and location of fluorine atoms in the molecular rigid core were doped (60 wt\% of each) in the same host mixture (HM) they produced two ferroelectric and two antiferroelectric liquid crystal mixtures [16,17]. Not only that, one of the dopant at lower concentration (10 wt $\%)$ produced an electroclinic mixture with large field-induced tilt and very small layer contraction [18] and at much higher concentration (75 wt\%), it formed a wide range ferroelectric mixture with a few hundred micro-second switching time [19]. Also, a different fluorinated chiral dopant with an oligomethylene spacer

was found to produce a ferroelectric mixture with much faster response time [20]. Even a binary mixture of one protonated oligomethylene spacer based chiral compound and one achiral biphenyl pyrimidine compound was found to exhibit room temperature ferroelectric mixture with moderate spontaneous polarization and sub-millisecond switching time [21]. Therefore, it might be inferred that the nature and concentration of the host and dopant influence the phase behavior and properties of the resulting mixtures enormously.

\subsection{Synchrotron Diffraction Study}

Mesophase structural investigation and temperature evolution of different phases of the mixtures were studied by synchrotron diffraction study. Transition 
temperatures were seen to be shifted to a higher temperature by $1-2^{\circ}$ than those observed from OPM and DSC study which probably resulted due to the use of different temperature baths. Very slow regulated cooling and planar polyimide coating of the inner surfaces of the cell resulted in an oriented sample. As shown in Fig. 3(a), in $\mathrm{SmC}^{*}$ phase, one very sharp low diffraction angle ring was observed characterizing one-dimensional positional ordering across the smectic layers. The sharpness of the ring slightly decreased in $\mathrm{SmA}^{*}$ phase and finally converted into a diffused ring in $\mathrm{N}^{*}$ phase (Fig. 3(b-c)). Temperature dependence of the layer spacings in smectic phases is shown in Fig. 4. It is observed that layer spacing in the $\mathrm{SmC}^{*}$ phase increases with increasing doping concentration. Layer spacings of the mixtures are also found to be almost linearly additive approaching towards the most extended length of the NMC $(32.41 \AA)$ obtained from the optimized geometry. From the figure, it is also noticed that the layer spacings increase with temperature, more in low concentration mixtures, signifying decrease of tilt angles of the molecules with respect to the layer normal which has been discussed further in Section 3.5.

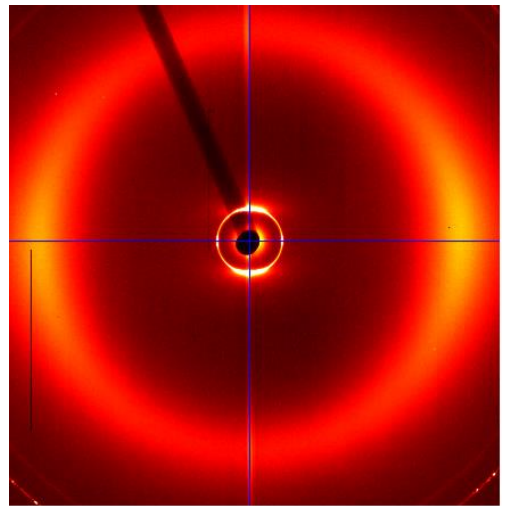

(a) $\mathrm{SmC}^{*}$

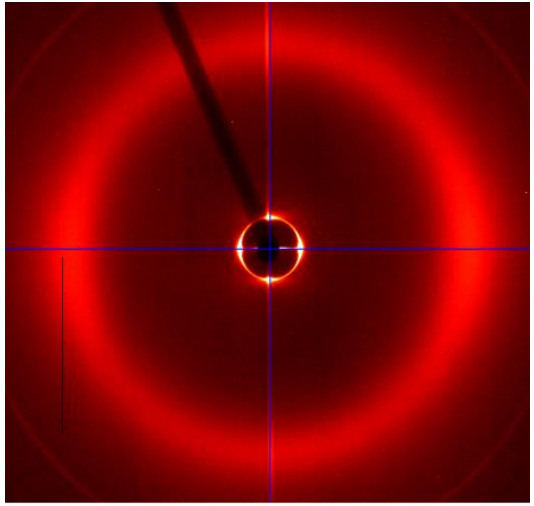

(b) $\mathrm{SmA}^{*}$

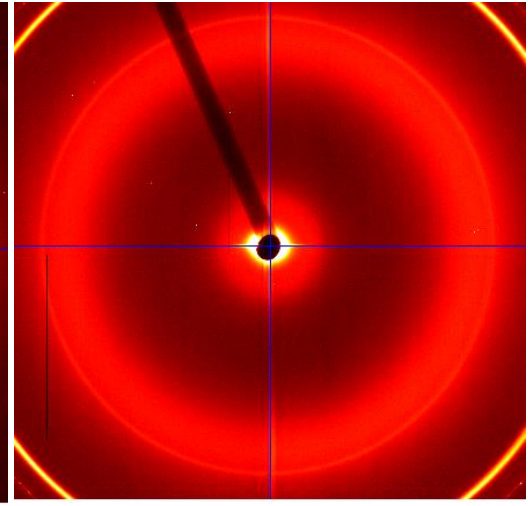

(c) $\mathrm{N}^{*}$

Fig. 3: Observed X-ray diffraction patterns of the mixture M2 


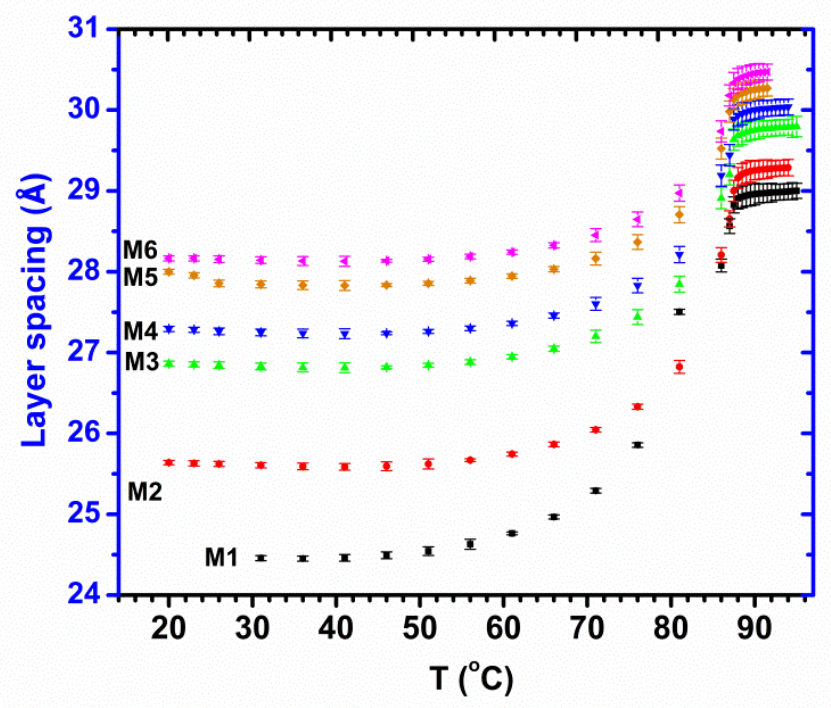

Fig. 4: Temperature variation of layer spacings of the mixtures.

\subsection{Dielectric Permittivity Study}

Transverse component of the real part of dielectric permittivity $\left(\varepsilon^{\prime}\right)$ in different phases for all the mixtures measured at $1 \mathrm{kHz}$ as a function of temperature are shown in Fig. 5. The dielectric behaviour of the three liquid crystalline phases, viz. $\mathrm{N}^{*}, \mathrm{SmA}^{*}$ and $\mathrm{SmC}^{*}$ can be clearly distinguished on the basis of the magnitude of $\varepsilon^{\prime}$. Except in the mixtures M1, M2, and M3, it has a very high magnitude in SmC* phase ( 4 for M1, 5 for M2, $~ 9$ for M3, 18 for M4, 45 for M5, $\sim 80$ for M6). Thus it is seen that the increase of doping concentration increases the magnitude of the real part of dielectric permittivity rapidly which is due to the increased polarity of the resulting mixture. The temperature dependence of $\varepsilon^{\prime}$ is also slightly different in the $\mathrm{SmC}^{*}$ phase. In $\mathbf{M 1}$ it decreases, in M2 it remains almost constant, in M3 and M4 it slightly increases and in M5 and M6 it slightly increases initially, then decreases. However, in all the mixtures near the $\mathrm{SmC}^{*}-\mathrm{SmA}^{*}$ transition temperature, $\varepsilon^{\prime}$ shows a diverging trend and on further heating, it abruptly falls to a value of $\sim 3$ in $\mathrm{SmA}^{*}$ phase. In M1 and M2, the magnitude of $\varepsilon^{\prime}$ of $N^{*}$ phase is found to be slightly higher than that of SmA* phase and shows a linearly increasing trend ( 2.9-3.4 for M1 and 
3.0-3.5 for M2) with temperature before going to the isotropic phase where it remains constant.
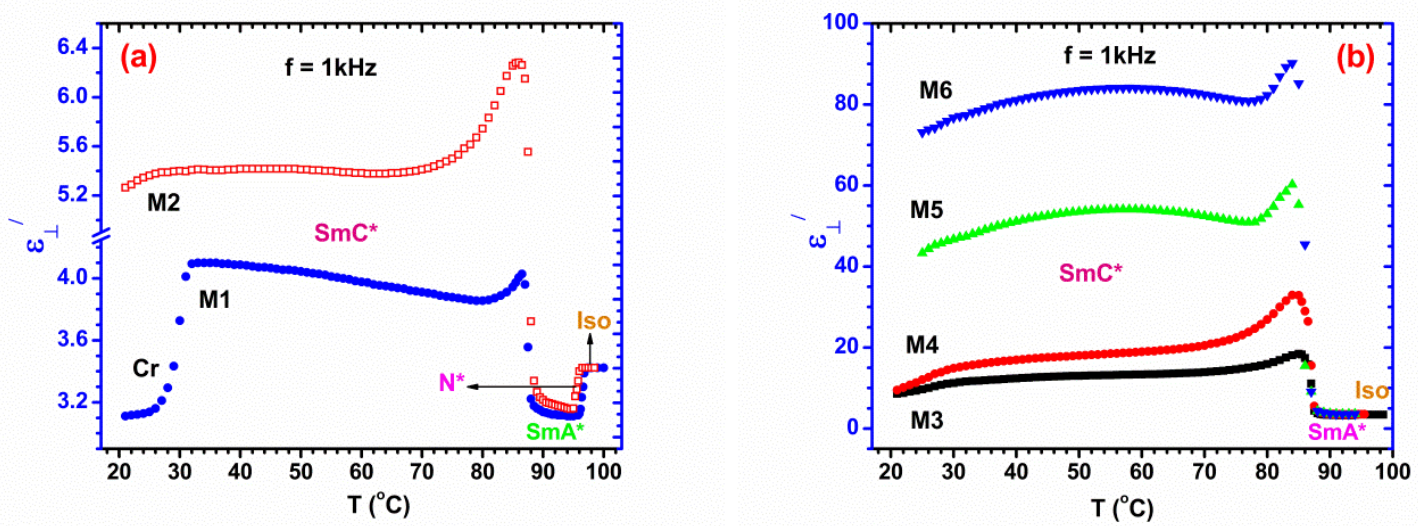

Fig. 5: Temperature variation of the real part of the dielectric permittivity $\left(\varepsilon^{\prime}\right)$ :

(a) M1, M2 and (b) M3, M4, M5 and M6

\subsection{Spontaneous Polarisation $\left(P_{S}\right)$ Study}

To test the growth of $\mathrm{P}_{\mathrm{S}}$ with the electric field it was measured as a function of the electric field at $20^{\circ} \mathrm{C}$ (only for $\mathbf{M 1}$ at $\mathrm{T}=32^{\circ} \mathrm{C}$ ). From this measurement, we calculated the saturation electric field $\left(\mathrm{E}_{\mathrm{sat}}\right)$, the field at which $\mathrm{P}_{\mathrm{S}}$ become $90 \%$ of its maximum. It is seen from Fig. 6(a-c) that the $\mathrm{E}_{\text {sat }}$ values for the mixtures M1-M6 are 12.5, 6.4, 5.2, 4.4, 4.2 and $4.0 \mathrm{~V} / \mu \mathrm{m}$, respectively. Thus the $\mathrm{E}_{\text {sat }}$ values are found to decrease with doping concentration which may be due to the fact that with increased concentration polarity of the mixtures increases and hence less electric field is required to align the dipoles.
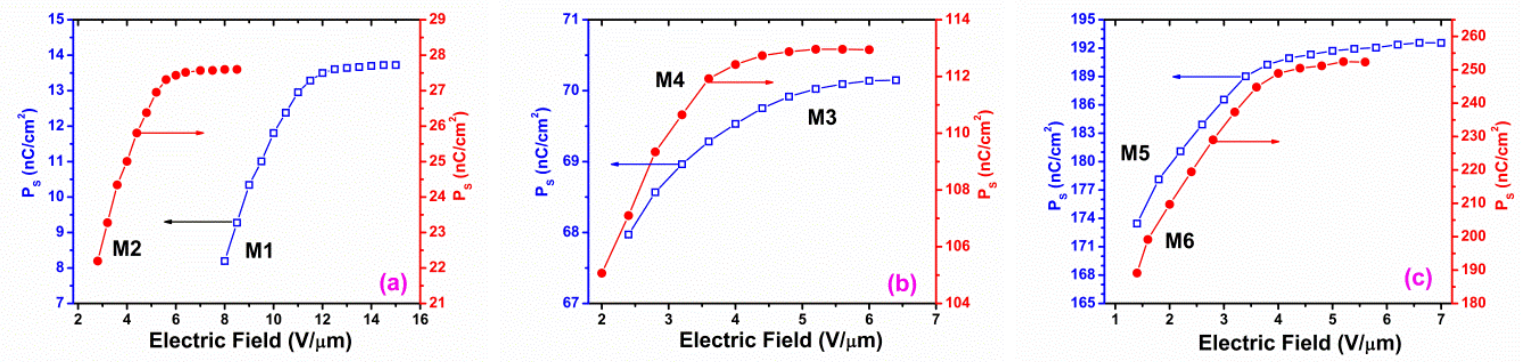

Fig. 6: Variation of spontaneous polarisation $\left(\mathrm{P}_{\mathrm{S}}\right)$ with electric field: (a) M1, M2; (b) M3, M4 and (c) M5, M6 

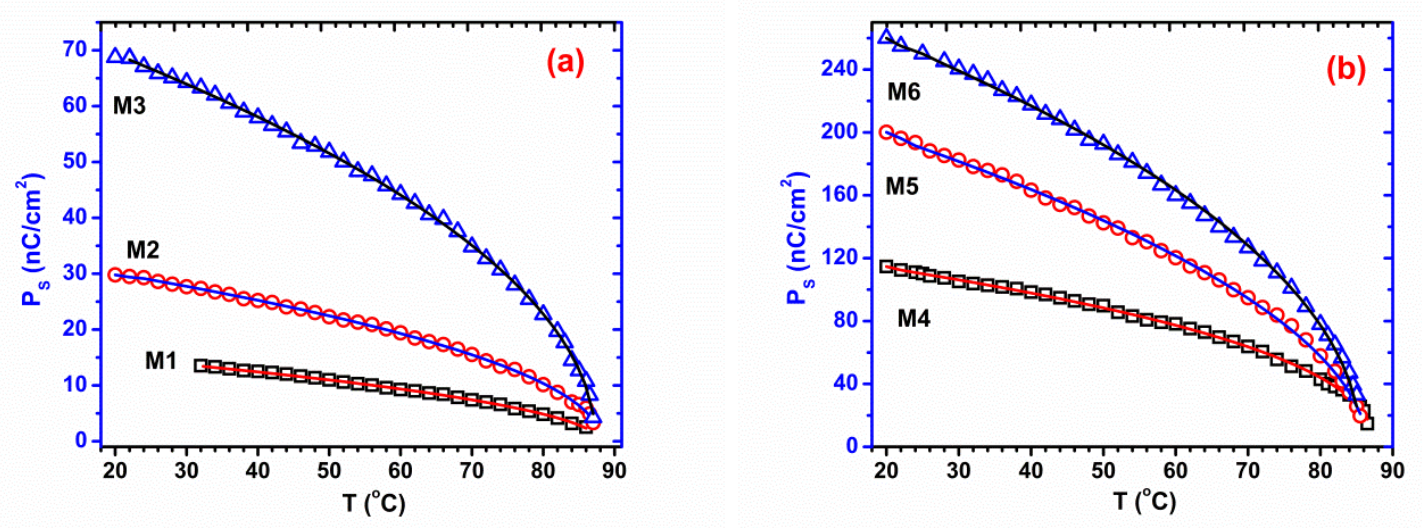

Fig. 7: Temperature variation of spontaneous polarisation $\left(\mathrm{P}_{S}\right)$ : (a) M1, M2, M3 and (b) M4, M5, and M6. Mean field fitted curves are shown as solid lines.

Temperature dependence of spontaneous polarisations $\left(\mathrm{P}_{\mathrm{S}}\right)$ measured at $\mathrm{E}_{\mathrm{sat}}$ is presented in Fig. 7. It is observed that the nature of variation of $P_{S}$ with temperature is similar for all the mixtures, Ps decreases with increasing temperature as expected. But with doping concentration, $\mathrm{P}_{\mathrm{S}}$ increases almost linearly: maximum values of $\mathrm{P}_{\mathrm{S}}$ in the mixtures $\mathbf{M 1}$ to $\mathbf{M 6}$ are found to be about 14, 30, 70, 120, 200 and $260 \mathrm{nC} / \mathrm{cm}^{2}$, respectively. Thus low dopant concentration induces less polarity in the mixtures compared to the higher dopant concentration as was revealed in the dielectric study. From the application point of view, moderate values of $P_{S}$ (about $50-150 \mathrm{nC} / \mathrm{cm}^{2}$ ) are required because high values of $\mathrm{P}_{\mathrm{S}}$ build up a large internal field in the devices that make polarisation reversal difficult and low values of $P_{S}$ require high electric field for reorientation, both of which are undesirable [22]. Thus so far $\mathrm{P}_{\mathrm{S}}$ is concerned moderate concentration mixtures (10-15\%) are more effective.

$\mathrm{P}_{\mathrm{S}}$ data are found to fit nicely to the mean-field model; the fitted parameters are listed in Table 4. From the table, one observes that the fitted $\mathrm{T}_{\mathrm{C}}$ values are very close to the actual $T_{C}$ and fitted $\beta$ values are close to the critical exponent value (0.5) for a second-order phase transition according to mean-field model [23]. It seems that the nature of the $\mathrm{SmC}^{*}-\mathrm{SmA}^{*}$ transition is second order. Very small 
enthalpy change observed in DSC study even at the heating rate of $5 \% \mathrm{~min}$ is an indication of this type of phase change. However, discontinuous change in tilt angle, more prominent in optical tilt (discussed in the next section), does not support this observation.

Table 4. Comparison of fitting parameters of $\mathrm{P}_{\mathrm{S}}$

\begin{tabular}{lccc}
\hline Mixture & Fitted $_{\mathrm{C}}\left({ }^{\circ} \mathrm{C}\right)$ & Actual $\mathrm{T}_{\mathrm{C}}\left({ }^{\circ} \mathrm{C}\right)$ & $\beta$ (Critical exponent) \\
\hline M1 & $88.02 \pm 0.21$ & 88.0 & $0.520 \pm 0.006$ \\
M2 & $88.11 \pm 0.13$ & 87.8 & $0.500 \pm 0.005$ \\
M3 & $87.36 \pm 0.07$ & 87.5 & $0.502 \pm 0.003$ \\
M4 & $87.37 \pm 0.13$ & 87.4 & $0.482 \pm 0.005$ \\
M5 & $86.48 \pm 0.11$ & 87.3 & $0.530 \pm 0.005$ \\
M6 & $85.61 \pm 0.16$ & 85.6 & $0.492 \pm 0.002$ \\
\hline
\end{tabular}

\subsection{Tilt Angle ( $\theta)$ Study}

The tilt angle of the molecules determined by electro-optical and X-ray scattering methods are presented in Fig. 8 and Fig. 9. The figures show that the general behavior of the tilt angle with temperature is the same for all the mixtures. An increase of the amount of the dopant NMC causes a decrease in the magnitude of the tilt of the mixtures. This decrease in primary order parameter (tilt angle) was also reflected in the thermal range of the $\mathrm{SmC}^{*}$ phase which was confirmed by OPM and DSC study, as the dopant concentration increased $\mathrm{SmC}^{*}{ }_{-} \mathrm{SmA}^{*}$ transition temperature decreased (see Table 4 and Fig. 1). However, the maximum values of the optical tilt angles for the mixtures M1 to M6 are found to be about $34^{\circ}, 30^{\circ}, 26^{\circ}, 25^{\circ}, 23.5^{\circ}$ and $22.5^{\circ}$, respectively. Optical tilt being close to $22.5^{\circ}$, the higher concentration mixtures (M5 and M6) are, therefore, expected to be suitable for SSFLCDs [22]. They also show 
relatively less temperature dependence which means contrast and brightness of the display devices will be less temperature dependent.
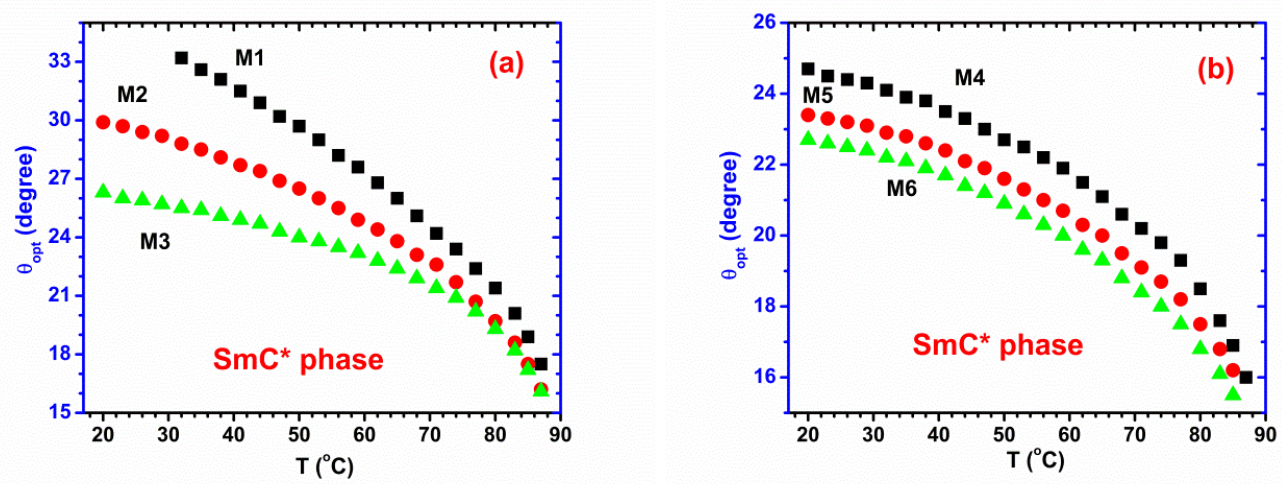

Fig. 8: Temperature variation of the optical tilt angle $\left(\theta_{\text {opt }}\right)$ : (a) M1, M2, M3 and (b) M4, M5, and M6

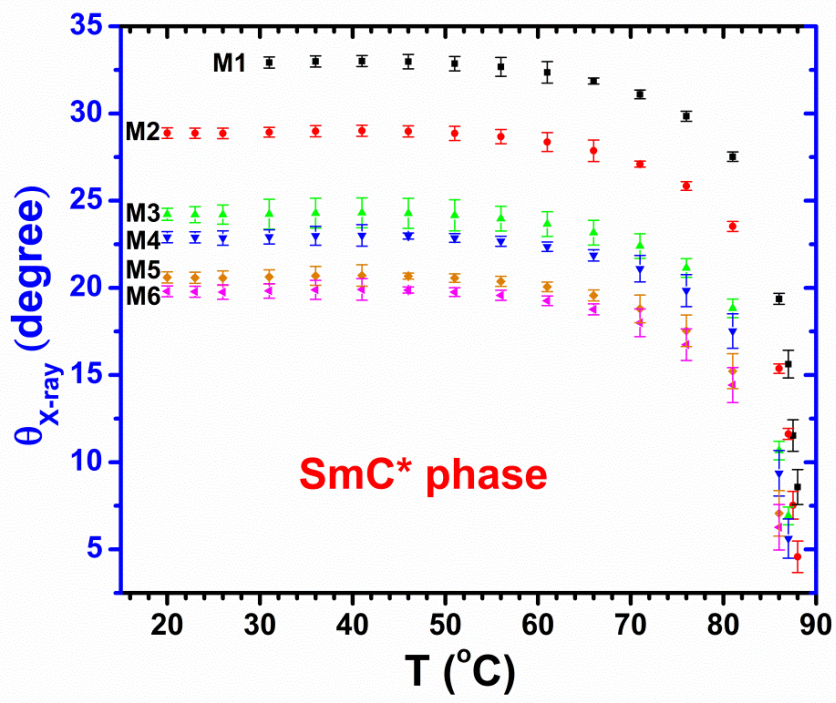

Fig. 9: Temperature variation of the $X$-ray tilt angle $\left(\theta_{\mathrm{X} \text {-ray }}\right)$ of the mixtures in SmC* phase

It is further noticed that although both the X-ray tilt $\left(\theta_{\mathrm{X} \text {-ray }}\right)$ and optical tilt $\left(\theta_{\mathrm{opt}}\right)$ show similar temperature dependence, but X-ray tilt is slightly smaller than the optical tilt. This is because X-ray tilt is a result of the tilt of electron density function of the whole molecule whereas optical tilt arises due to the tilt of only the rigid polarisable group of the molecule. Since the host and the dopant 
molecules possess large flexible parts, X-ray tilt may be less than the optical tilt $[24,25]$. However, $\theta_{\text {opt }}$ is more useful from the application point of view.

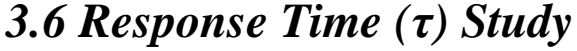

Observed temperature dependence of the response time of the mixtures is shown in Fig. 10. From the figure it is clear that all the mixtures show a similar type of temperature dependence, in low-temperature region $\tau$ decreases very quickly, then the rate of decrement falls off and finally, at high temperatures, it becomes almost constant. It is further observed that the magnitude of $\tau$ decreases with doping concentration: the maximum value of $\tau$ in the mixtures

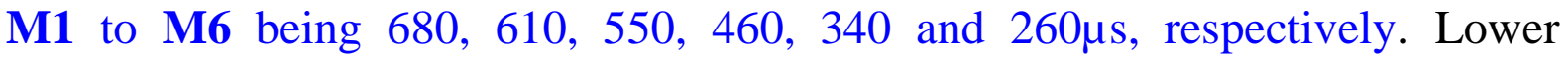
response time is desired from the application point of view because lower is the response time faster will be the electro-optical response of the device. Therefore, with respect to the response speeds higher dopant concentration mixtures (M5 and M6) are suitable.
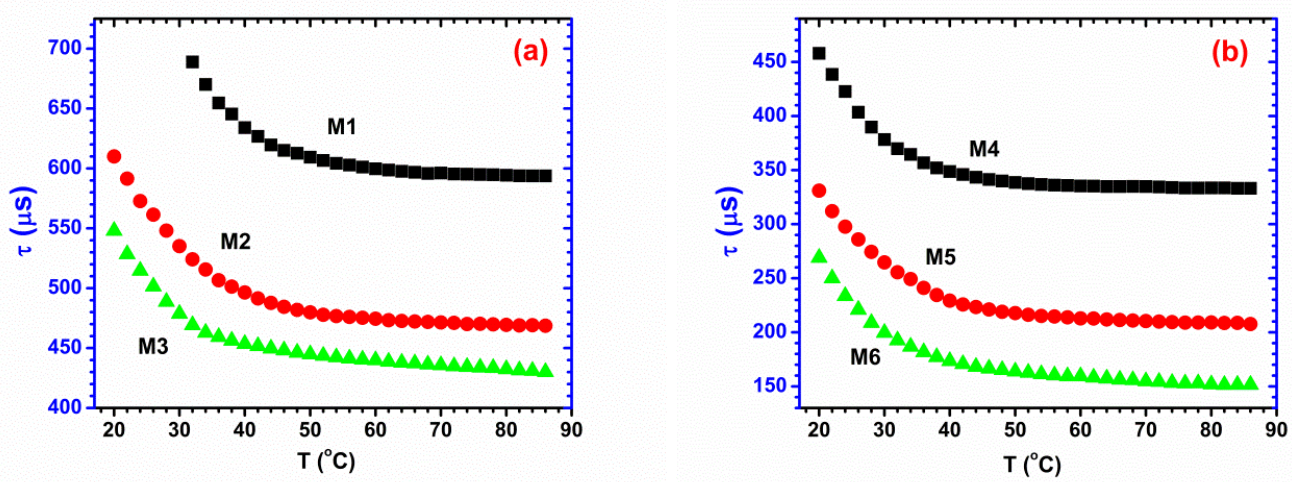

Fig. 10: Temperature variation of response time ( $\tau$ ): (a) M1, M2, M3 and (b) M4, M5, and M6

\subsection{Rotational Viscosity $\left(\gamma_{\varphi}\right)$ and Activation Energy $\left(E_{a}\right)$ Study}

The rotational viscosity $\left(\gamma_{\varphi}\right)$, related to the rotations of the molecules about the Smectic C cone, is an important parameter of the $\mathrm{SmC}^{*}$ phase, which strongly influences the switching time between the field-induced states of FLCs.Temperature dependence of $\gamma_{\varphi}$ is presented in Fig. 11. Rotational 
viscosity is found to increase linearly with concentration. In all the mixtures, at low temperature $\gamma_{\varphi}$ exhibits relatively high values $\left(1.118-3.032 \mathrm{~N}-\mathrm{sec} / \mathrm{m}^{2}\right)$ and thereafter decreases rapidly with increasing temperature. Applying the Arrhenius law to viscosity data [26], the activation energies $\left(E_{a}\right)$ were calculated. In the mixtures M1 to M6 the $\mathrm{E}_{\mathrm{a}}$ values are found to be 13.42, 14.65, $15.72,19.22,19.65$ and $19.94 \mathrm{~kJ} / \mathrm{mol}$, respectively. Therefore, activation energy also increases with increasing concentration.
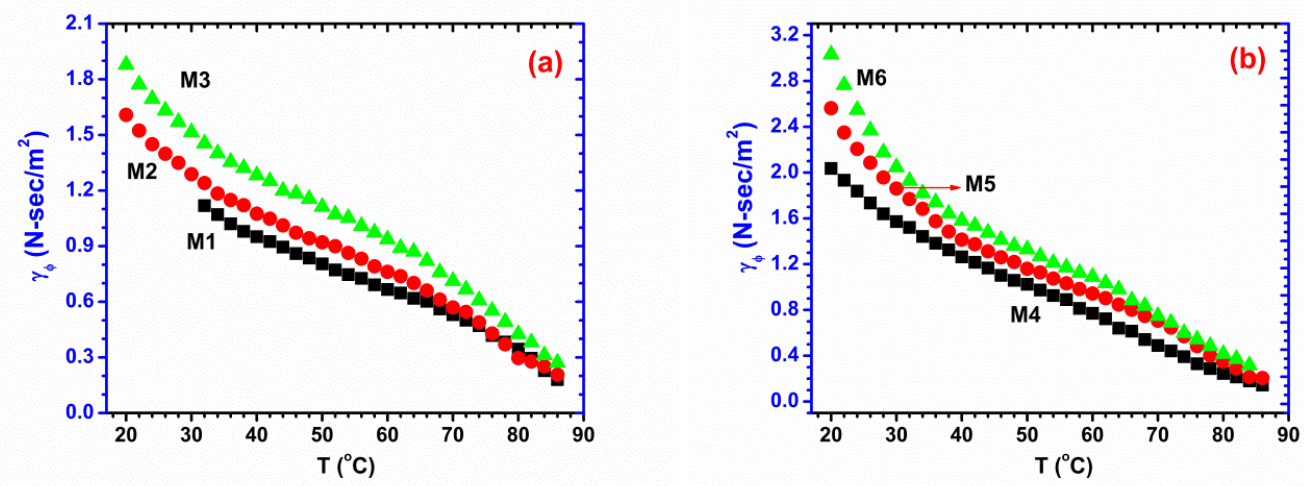

Fig. 11: Temperature variation of rotational viscosity $\left(\gamma_{\varphi}\right)$ : (a) M1, M2, M3 and (b) M4, M5, and M6

For ease of comparison, various parameters important for display application have been listed in Table 5.

Table 5. Comparison of display parameters of the formulated mixtures at $20^{\circ} \mathrm{C}^{\#}$

\begin{tabular}{lccccccc}
\hline Mixture & $\mathrm{T}_{\mathrm{C}^{*}-\mathrm{T}_{\mathrm{A}^{*}}\left({ }^{\circ} \mathrm{C}\right)}$ & $\varepsilon^{\prime}$ & $\mathrm{P}_{\mathrm{S}}\left(\mathrm{nC} / \mathrm{cm}^{2}\right)$ & $\theta_{\mathrm{opt}}\left({ }^{\circ}\right)$ & $\tau(\mu \mathrm{s})$ & $\gamma_{\varphi}\left(\mathrm{N}-\mathrm{s} / \mathrm{m}^{2}\right)$ & $\mathrm{E}_{\mathrm{a}}(\mathrm{kJ} / \mathrm{mol})$ \\
\hline M1 & $32-88$ & 4.0 & 13 & 33.2 & 690 & 1.118 & 13.42 \\
M2 & $<12-87.8$ & 5.3 & 30 & 29.9 & 610 & 1.608 & 14.65 \\
M3 & $<12-87.5$ & 8.6 & 70 & 26.3 & 550 & 1.879 & 15.72 \\
M4 & $<12-87.4$ & 9.5 & 110 & 24.7 & 460 & 2.035 & 19.22 \\
M5 & $<12-86.3$ & 43.3 & 195 & 23.4 & 330 & 2.562 & 19.65 \\
M6 & $<12-85.6$ & 73.1 & 245 & 22.7 & 260 & 3.032 & 19.94 \\
\hline
\end{tabular}

${ }^{\#}$ parameters of $\mathbf{M} 1$ at $32^{\circ} \mathrm{C}$. 


\section{Conclusions}

By varying concentration of a chiral terphenylate dopant into an achiral host matrix we have prepared six multi-component ferroelectric liquid crystal mixtures. Though the dopant is non-mesogenic in character, its mixtures in the multi-component host matrix exhibit a very wide range of ferroelectric $\mathrm{SmC}^{*}$ phase starting from below room temperature, at least from $12^{\circ} \mathrm{C}$. Various physical properties of the mixtures are found to be very sensitive to dopant concentration. With respect to the magnitude of different display parameters such as temperature range, dielectric permittivity, spontaneous polarization, optical tilt angle, rotational viscosity and response time the mixtures with moderate concentrations will be most suitable for SSFLC based applications.

\section{Acknowledgments}

We thankfully acknowledge the financial support under BRNS (DAE) project 2010/37P/44/BRNS/1441. Portions of this research were carried out at the light source PETRA III of DESY, a member of Helmholtz Association (HGF). Financial support by the Department of Science and Technology (Government of India) provided within the framework of the India@DESY collaboration is gratefully acknowledged. We are also grateful to Professor Roman Dabrowski, MUT, Poland for supplying the compounds.

\section{References}

[1] M. E. Lines and A. M. Glass. "Principles and Applications of Ferroelectric and Related Materials”. Oxford University Press, Oxford, 2001.

[2] L. Pardo and J. Ricote. "Multifunctional Polycrystalline Ferroelectric Materials”. Springer, Dordrecht, 2011.

[3] R. B. Meyer, L. Liebert, L. Strzelecki, et al. Ferroelectric Liquid Crystals. Phys. Lett. (Paris). 1975; 36: 69-71. 
[4] R. B. Meyer. Ferroelectric Liquid Crystals: A Review. Mol. Cryst. Liq. Cryst., 1977; 40: 33-38.

[5] N. A. Clark and S. T. Lagerwall. Sub-microsecond bistable electro-optic switching in liquid crystals. Appl Phys Lett., 1980; 36: 899-902.

[6] W. Kuczynski and H. Stegemeyer. Ferroelectric properties of smectic C liquid crystals with an induced helical structure. Chem. Phys. Lett., 1980; 70: 123-126.

[7] M. Wand, R. Vobra, W. Thurmes, et al. New ferroelectric liquid crystal host materials for use in optoelectronic applications. Proc SPIE, 1994; 2175: 1-6.

[8] W. Thurmes, M. Wand, R. Vohra, et al. FLC materials form microdisplay applications. Proc SPIE, 1997; 3015: 1-6.

[9] M. Wand, W. Thurmes, M. Meadows, et al. FLC displays for high resolution magnified view and projection applications. Proc SPIE, 1999; 3635: 1-6.

[10] N. Bennis, A. Spadlo, R. Dabrowski, et al. Non-conventional alignment surfaces for antiferroelectric liquid crystals. Mol. Cryst. Liq. Cryst., 2004; 422: $37-45$.

[11] K Miyasato, S Abe, H Takezoe, et al. Direct Method with Triangular Waves for Measuring Spontaneous Polarization in Ferroelectric Liquid Crystals. Jpn J App Phys., 1983; 22: L661-L663.

[12] P. G. de Gennes. "The physics of liquid crystals". oxford univ. press: Oxford, 1975.

[13] V. A. Baikalov, L. A. Beresnev, and L. M. Blinov. Measures of the molecular tilt angle and optical anisotropy $\mathrm{n}$ ferroelectric liquid crystals. Mol. Cryst. Liq. Cryst., 1985; 127: 397-406.

[14] E. P. Pozhidaev, M. A. Osipov, V. Chigrinov, et al. Rotational viscosity of the smectic C* phase of ferroelectric liquid crystals. Sov. Phys. JETP., 1988; 94: $125-132$.

[15] I. Dierking. Textures of liquid crystals. WILEY-VCH: 2003. 
[16] A. Debnath and P. K. Mandal. Effect of fluorination on the phase sequence, dielectric and electro-optical properties of ferroelectric and antiferroelectric mixtures. Liq. Cryst., 44; 2017: 2192-2202.

[17] A. Debnath and P. K. Mandal. Dielectric properties of four room temperature ferroelectric and antiferroelectric multi-component liquid crystalline mixtures. Liq. Cryst., Published online; 2017. doi: 10.1080/02678292.2018.1489986

[18] A. Debnath, D. Sinha, and P. K. Mandal. Wide range room temperature electroclinic liquid crystal mixture with large induced tilt and very small layer contraction. J App. Phy., 119; 2016: 124103.

[19] A. Debnath, D. Sinha, P. K. Mandal, and R. Dabrowski. Formulation of a room temperature ferroelectric liquid crystal mixture with sub-millisecond switching time. AIP Conf. Proc., 1665; 2015: 040004 (1-3).

[20] A. Debnath and P. K. Mandal. Wide range room temperature ferroelectric liquid crystal mixture with microsecond order switching. J Mol. Liq., 221; 2016: 287-291.

[21] A. Debnath, P. K. Mandal, D. Wegłowska and R. Dabrowski. Induction of a room temperature ferroelectric $\mathrm{SmC}^{*}$ phase in binary mixtures with moderate spontaneous polarization and sub-millisecond switching time. RSC Advances, 6; 2016: 84369-84378.

[22] M. Hird. Ferroelectricity in liquid crystals-materials, properties, and applications. Liq. Cryst., 2011; 38: 1467-1493.

[23] J. Zubia, M. Castro, J. A. Puertolas, et al. Character of the smectic- $A$ chiral-smectic- $C$ phase transition near a chiral-nematic-smectic- $A$-chiralsmectic-C point. Phys. Rev. E., 1993; 48: 1970-1976.

[24] R. Bartolino, J. Doucet, and G. Durand. Molecular tilt in the smectic-C phase-zigzag model. Ann. de Phys., 1978; 3: 389-395.

[25] M. S. Spector, P. A. Heiney, J. Naciri, et al. Electroclinic liquid crystals with large induced tilt angle and small layer contraction. Phys. Rev. E., 2000; 61: 1579-1583. 
[26] J. Hemine, C. Legrand, A. Daoudi, et al. Electro-optical and dielectric characterizations of the Goldstone mode relaxation in ferroelectric chiral smectic C liquid crystals. J. Phys. Cond. Matt., 2007; 19: 296203(1-10). 